\title{
The prognostic factors of survival and recurrence in patients with serous ovarian and uterine cancers treated in a single institution for $\mathbf{1 7}$ years Czynniki prognostyczne przeżycia i wznowy u chorych na surowiczego raka jajnika i trzonu macicy leczonych w jednym ośrodku przez okres 17 lat
}

\section{Oncology Clinic, Tepecik Research and Education Hospital, İzmir, Turkey}

Correspondence: Gülşą Selvi Demirtaş, MD, Department of Gynecology and Obstetrics, Tepecik Research and Education Hospital, Gaziler Cd, No: 468, 35120, İzmir, Turkey, tel.: (+90) 0533727 15 12, fax: (+90) 232457 9651, e-mail: drg.selvi@gmail.com

\begin{abstract}
Objective: In this study, we aimed to identify the prognostic factors of survival and recurrence in ovarian and uterine serous cancer patients. Materials and methods: This was a retrospective study conducted at Tepecik Research and Education Hospital, İzmir, Turkey, between January 2002 and January 2019. The medical files of 2,027 endometrial and 821 ovarian patients who underwent examination for endometrial cancer and epithelial ovarian cancer were examined retrospectively by the same author. The data of eligible 385 and 49 patients diagnosed with ovarian and uterine serous carcinoma, respectively, were identified for analysis from the hospital database. Descriptive, univariate, and multivariate Cox regression and binary logistic regression analyses of patients were performed. Results: The mean age of ovarian serous cancer patients $(n=385)$ was $53.9 \pm 10.9$ years. The mean age of uterine serous cancer patients $(n=49)$ was $67.2 \pm 10.6$ years. A total of 81 ovarian serous cancer patients $(21.0 \%)$ had stage 1 , while $24(6.2 \%)$ had stage 2 , and $31(8.1 \%)$ had stage 4 disease. A total of 26 uterine serous carcinoma patients $(53.1 \%)$ had stage 1 disease, $6(12.2 \%)$ had stage 2, 10 (20.4\%) had stage 3 , and $7(14.3 \%)$ had stage 4 disease. For ovarian serous patients, stage, grade, optimality, neoadjuvant chemotherapy, adjuvant chemotherapy cycle number, and recurrence had impact on both overall and disease-free survival $(p<0.05)$. For uterine serous cancer patients, optimality was the only prognostic factor for both survival and recurrence $(p=0.01$ and $p=0.01$, respectively). Conclusion: In ovarian serous cancer patients, we found that disease stage, grade, optimality, neoadjuvant chemotherapy, and adjuvant chemotherapy cycle number had impact on overall and disease-free survival in both univariate and multivariate Cox regression analysis, whereas disease stage and optimality were the only significant prognostic factors for recurrence in ovarian serous cancer patients. However, in patients with uterine serous carcinomas, optimal surgery was the only independent prognostic factor both for survival and recurrence.
\end{abstract}

Keywords: serous carcinoma, endometrial cancer, ovarian epithelial cancer

Streszczenie Cel: Celem badania było określenie czynników prognostycznych przeżycia i wznowy u chorych na surowiczego raka jajnika i trzonu macicy. Materiał i metody: Retrospektywne badanie wykonano w Szpitalu Klinicznym Tepecik (İzmir, Turcja) w okresie od stycznia 2002 do stycznia 2019 roku. Analizą retrospektywną przeprowadzoną przez tego samego autora objęto dokumentację medyczną 2027 pacjentek z rakiem trzonu macicy i 821 pacjentek z rakiem jajnika. Ze szpitalnej bazy danych pozyskano dane 385 i 49 kwalifikujących się pacjentek z rozpoznaniem odpowiednio raka surowiczego jajnika i raka surowiczego trzonu macicy. Do analizy danych pacjentów wykorzystano analizę opisową, jednowymiarową i wielowymiarową regresji Coxa oraz binarną regresję logistyczną. Wyniki: Średnia wieku pacjentek z surowiczym rakiem jajnika $(n=385)$ wyniosła 53,9 $\pm 10,9$ roku, a średnia wieku pacjentek z surowiczym rakiem trzonu macicy $(n=49)-67,2 \pm 10,6$ roku. Pierwszy, drugi i czwarty stopień zaawansowania raka surowiczego jajnika stwierdzono odpowiednio u 81 (21,0\%), 24 (6,2\%) i $31(8,1 \%)$ pacjentek. Pierwszy, drugi, trzeci i czwarty stopień zaawansowania raka surowiczego trzonu macicy odnotowano odpowiednio u $26(53,1 \%), 6$ (12,2\%), 10 (20,4\%) i 7 (14,3\%) pacjentek. W przypadku pacjentek z surowiczym rakiem jajnika czynniki takie jak stopień zaawansowania choroby, stopień złośliwości, zastosowanie optymalnego leczenia operacyjnego, zastosowanie chemioterapii neoadiuwantowej, liczba cykli chemioterapii adiuwantowej oraz wystąpienie wznowy wpływały zarówno na przeżycie ogólne, jak i przeżycie wolne od choroby $(p<0,05)$. W przypadku pacjentek z surowiczym rakiem trzonu macicy jedynym czynnikiem prognostycznym zarówno dla przeżycia, jak i wznowy okazało się optymalne leczenie 
operacyjne (odpowiednio $p=0,01$ i $p=0,01$ ). Wniosek: Zarówno w jednoczynnikowej, jak i wieloczynnikowej analizie regresji Coxa wykazano, że na ogólne przeżycie i przeżycie wolne od objawów choroby pacjentek z rozpoznaniem surowiczego raka jajnika wpływają takie czynniki, jak stopień zaawansowania choroby, stopień złośliwości, optymalność leczenia operacyjnego, zastosowanie chemioterapii neoadiuwantowej oraz liczba cykli chemioterapii adiuwantowej, przy czym stopień zaawansowania choroby i zastosowanie optymalnego leczenia operacyjnego stanowiły jedyne istotne czynniki prognostyczne wznowy u tych pacjentek. Natomiast w przypadku pacjentek z rakiem surowiczym trzonu macicy jedynym niezależnym czynnikiem prognostycznym zarówno dla przeżycia, jak i wznowy był optymalny zabieg operacyjny.

Słowa kluczowe: rak surowiczy, rak trzonu macicy, nabłonkowy rak jajnika

\section{INTRODUCTION}

S erous ovarian cancer, the most common and aggressive form of epithelial ovarian cancer, remains the leading cause of cancer-related death among all gynecologic cancers in the world ${ }^{(1,2)}$. This high mortality rate is explained in part by the advanced disease stage at the time of diagnosis, with approximately $75 \%$ of patients presenting with stage 3 or 4 disease ${ }^{(3)}$. Therapeutic strategies include cytoreductive surgery followed by 6 cycles of platinum- and taxane-based chemotherapy $(\mathrm{CT})^{(4,5)}$. Despite the fact that the majority of patients achieve complete clinical remission following this treatment regimen, the prognosis in ovarian cancer remains unfavorable, with a 5-year survival rate of approximately $50 \%{ }^{(5)}$.

In this study, we identified the prognostic factors which had impact on survival and recurrence in ovarian and uterine serous cancer patients.

\section{MATERIALS AND METHODS}

This was a retrospective study conducted at Tepecik Research and Education Hospital, İzmir, Turkey, between January 2002 and January 2019. The medical files of 2,027 endometrial and 821 ovarian patients who underwent examination for endometrium cancer and epithelial ovarian cancer were examined retrospectively by the same author. The data of eligible 385 and 49 patients diagnosed with ovarian and uterine serous carcinoma, respectively, were identified for analysis from the hospital database. A total of 385 ovarian and 49 uterine cancer patients with the final histopathological diagnosis of serous cancer were included, and 436 ovarian and 1,978 endometrial cancer patients with unavailable data and other histopathological diagnoses were excluded from the study. The patients' age, past medical history, gravida, parity, operation, pre- and postoperative histopathologic results, tumor grade, size, preoperative CA-125 levels, myometrial invasion (MI), pelvic (P), paraaortic (PA) lymph node (LN) metastases, lymphovascular space invasion (LVSI), stage of the disease, and adjuvant therapies were reviewed. The patients' pathology specimens were evaluated by expert pathologists. The patients were staged according to the FIGO 2009 staging system (between 2002 and 2009 stages were reevaluated according to the 2009 FIGO). The patients were stratified for neoadjuvant CT (NACT) followed by interval cytoreductive surgery and adjuvant $\mathrm{CT}$, and primary debulking surgery and adjuvant $\mathrm{CT}$ according to the $\mathrm{MD}$ Anderson Algorithm. Surgery in the form of total abdominal hysterectomy (TAH) and bilateral salpingooophorectomy (BSO), either with or without pelvic and paraaortic lymph node dissection (P PA LND), was performed in all the operable patients. Additionally, appendectomy (APP), omentectomy (OMM), splenectomy, pelvic peritonectomy, and implant resection were performed in cases where necessarily indicated. Optimal surgery was defined as having residual tumor nodules each measuring $1 \mathrm{~cm}$ or less in the maximum diameter. Overall survival (OS) was calculated from the date of diagnosis to the date of death or last follow-up. Diseasefree survival (DFS) was measured from the date of diagnosis to the date of first recurrence or progression of disease. Depending on clinical and pathological factors obtained from surgical staging, radiation therapy (RT) or CT was planned by members of a tumor board comprised of a pathologist, gynecological oncologist, radiation oncologist, and medical oncologist. Postoperative medical oncology and radiation oncology reports were also evaluated. Optimality was defined both by the surgeon's declaration and postoperative imaging. If there was a discrepancy between the imaging findings and the surgeon's statement, this was considered as suboptimal surgery. Adjuvant therapy including CT alone, RT including internal radiotherapy (IRT) and external radiotherapy (ERT) alone, or a combination of both, was administered to the patients based on disease stage, age, nodal metastasis status, performance status, and the presence/absence of medical comorbidities. The external beam radiation dose was 45-50 Gy with conventional fractionation. The dose of intravaginal brachytherapy was prescribed at a depth of $0.5 \mathrm{~cm}$ from the surface of ovoids. Chemotherapy regimens were administered intravenously every 21 days to a maximum of 6 cycles. Statistical analyses were performed using IBM SPSS Statistics 22.0 (SPSS Inc., Chicago, IL). Shapiro-Wilk's test, histograms, and Q-Q plots were used to identify whether the data was normally distributed or not. The descriptive statistics were defined as median \pm standard deviation and median (minimum and maximum values). The chi-square test (Pearson's chi-square and Pearson's exact chi-square tests) was used to compare the proportions between groups. Univariate and multivariate Cox regression models were 
used to identify factors that affected the OS and DFS. Binary logistic regression analysis with the enter method were used to identify the prognostic factors for disease recurrence. Beta coefficients, $p$ values, and odds ratios were identified.

\section{RESULTS}

The mean age of ovarian serous cancer patients $(n=385)$ was $53.9 \pm 10.9$ years. Most patients had stage 3 disease $[n=249$ (64.7\%), 95\% confidence interval (CI): 60.0-69.3]. A total of 81 patients (21.0\%) (95\% CI: 17.2-25.1) had stage 1, while 24 (6.2\%) (95\% CI: 3.8-8.9) had stage 2, and 31 patients (8.1\%) (95\% CI: $5.4-10.7)$ had stage 4 disease. A total of 79 patients (20.5\%) had grade 1, and $122(31.7 \%)$ and 184 $(47.8 \%)$ had grade 2 and 3 disease, respectively. The mean tumor size was $31.3 \pm 40.5 \mathrm{~mm}$. One hundred and eighty patients had cancer antigen 125 (CA-125) \500, and 205 patients had CA- $125 \geq 500$. Twelve patients (3.1\%) underwent TAH BSO. A total of 94 (24.4\%) (95\% CI: 19.8-28.7) patients had TAH BSO P PA LND, and 38 patients (9.9\%) (95\% CI: 7-13.1) underwent TAH BSO P LND. Thirty patients (7.8\%) (95\% CI: 5.0-10.6) underwent TAH BSO and OMM, while 36 patients (9.4\%) (95\% CI: 6.4-12.3) had TAH BSO OMM and APP. A total of 34 patients (8.8\%) (95\% CI: 6.1-11.9) were treated by TAH BSO OMM P LND APP, and 100 patients (26.0\%) (95\% CI: 21.6-30.3) underwent TAH BSO OMM P PA LND APP. Three patients ( $0.8 \%)$ received hyperthermic intraperitoneal chemotherapy (HIPEC), while 32 patients (8.3\%) (95\% CI: 5.6-11.2) underwent TAH BSO OMM P PA LND APP and implant excision. Three patients $(0.8 \%)$ (95\% CI: 0.0-1.8) who underwent surgery were evaluated as inoperable and received NACT followed by debulking surgery (TAH BSO OMM P PA LND). Overall, 63.6\% $(n=245)$ patients had optimal surgery. A total of 109 patients (28.3\%) (95\% CI: 23.8-32.9) received NACT. The mean CT cycle number was $6.19 \pm 2.03$. In 110 patients (28.6) (95\% CI: 24.1-33.2), recurrence was observed. The mean OS and DFS was $47.0 \pm 36.3$ months and $45.1 \pm 34.7$ months, respectively (Tab. 1). In univariate analysis, disease stage, grade, CA-125, ascites, optimality, NACT, adjuvant CT cycle number, and recurrence had impact both on the DFS and OS. Tumor size had an effect only on the OS in univariate analysis. In multivariate Cox regression analysis, disease stage, grade, optimality, NACT, adjuvant CT cycle number and recurrence were found to have impact both on the OS and DFS. CA-125 and ascites had no significant effect on the OS and DFS in multivariate analysis. Similarly, tumor size had impact only on the OS in multivariate analysis (Tab. 2). In binary logistic regression analysis, disease stage and suboptimality were shown to be significant prognostic factors for recurrence (odds ratio: 2.9 and 2.4, $p=0.00$ and $p=0.00$, respectively) (Tab. 3).

In uterine serous carcinoma patients $(n=49)$, the mean age was $67.2 \pm 10.6$ years. Most patients had stage 1 disease $[n=26(53.1 \%)]$. Six patients $(12.2 \%)$ had stage 2 , while

\begin{tabular}{|c|c|c|}
\hline & $n(\%)$ & $95 \% \mathrm{Cl}$ \\
\hline \multicolumn{3}{|l|}{ Stage } \\
\hline 1 & $81(21.0 \%)$ & $17.2-25.1$ \\
\hline 2 & $24(6.2 \%)$ & $3.8-8.9$ \\
\hline 3 & $249(64.7 \%)$ & $60.0-69.3$ \\
\hline 4 & $31(8.1 \%)$ & $5.4-10.7$ \\
\hline \multirow[t]{2}{*}{ Age } & Mean & $\pm S D$ \\
\hline & 53.96 & 10.9 \\
\hline Grade & $n(\%)$ & $95 \% \mathrm{Cl}$ \\
\hline 1 & $79(20.5 \%)$ & $16.4-24.8$ \\
\hline 2 & $122(31.7 \%)$ & $27.1-36.9$ \\
\hline 3 & $184(47.8 \%)$ & $42.6-52.7$ \\
\hline CA-125 & $n(\%)$ & $95 \% \mathrm{Cl}$ \\
\hline$<500$ & $180(46.8 \%)$ & $2.5-41.9$ \\
\hline$\geq 500$ & $205(53.2 \%)$ & $2.5-48.5$ \\
\hline \multirow[t]{2}{*}{ Tumor size } & Mean & $\pm S D$ \\
\hline & 31.3 & 40.5 \\
\hline Ascites & $n(\%)$ & $95 \% \mathrm{Cl}$ \\
\hline$<500$ & $168(43.6 \%)$ & $38.9-48.8$ \\
\hline$\geq 500$ & $217(56.4 \%$ & $51.2-61.1$ \\
\hline OP & $n(\%)$ & $95 \% \mathrm{Cl}$ \\
\hline TAH BSO & $12(3.1 \%)$ & $1.5-4.9$ \\
\hline TAH BSO OMM & $30(7.8 \%)$ & $5.0-10.6$ \\
\hline TAH BSO OMM PLND & $38(9.9 \%)$ & $7.0-13.1$ \\
\hline TAH BSO OMM PPLND & $94(24.4 \%)$ & $19.8-28.7$ \\
\hline TAH BSO OMM APP & $36(9.4 \%)$ & $6.4-12.3$ \\
\hline TAH BSO OMM PLND APP & $34(8.8 \%)$ & $6.1-11.9$ \\
\hline TAH BSO OMM PPLND APP & $100(26.0 \%)$ & $21.6-30.3$ \\
\hline TAH BSO OMM PPLND APP HIPEC & $3(0.8 \%)$ & $0.0-1.8$ \\
\hline TAH BSO OMM PPLND APP Splenectomy & $3(0.8 \%)$ & $0.0-1.7$ \\
\hline TAH BSO OMM PPLND APP Implant excision & $32(8.3 \%)$ & $5.6-11.2$ \\
\hline Only BX & $3(0.8 \%)$ & $0.0-1.8$ \\
\hline \multicolumn{3}{|l|}{ Optimality } \\
\hline Optimal & $245(63.6 \%)$ & $58.9-68.7$ \\
\hline Suboptimal & $140(36.4 \%)$ & $31.3-41.1$ \\
\hline \multicolumn{3}{|l|}{ NACT } \\
\hline No & $276(71.7 \%)$ & $67.1-76.2$ \\
\hline Yes & $109(28.3 \%)$ & $23.8-32.9$ \\
\hline \multirow[t]{2}{*}{ CT cycle number } & Mean & $\pm S D$ \\
\hline & 6.19 & 2.03 \\
\hline Recurrence & $n(\%)$ & $95 \% \mathrm{Cl}$ \\
\hline No & $275(71.4 \%)$ & $66.8-75.9$ \\
\hline Yes & $110(28.6 \%)$ & $24.1-33.2$ \\
\hline \multirow[t]{2}{*}{ DFS } & Mean & $\pm S D$ \\
\hline & 45.1 & 34.7 \\
\hline \multirow[t]{2}{*}{ OS } & Mean & $\pm S D$ \\
\hline & 47.0 & 36.3 \\
\hline \multicolumn{3}{|c|}{$\begin{array}{l}\text { 95\% CI - 95\% confidence interval; APP - appendectomy; BX - biopsy; } \\
\text { CA-125 - cancer antigen 125; CI - chemotherapy; DFS - disease-free survival; } \\
\text { HIPEC - hyperthermic intraperitoneal chemotherapy; LVSI - lymphovascular } \\
\text { space invasion; NACT - neoadjuvant chemotherapy; OMM - omentectomy; } \\
\text { OP - operation; OS - overall survival; PLND - pelvic lymph node dissection; } \\
\text { PPLND - pelvic paraaortic lymph node dissection; RT - radiotherapy; } \\
\text { TAH BSO - total abdominal hysterectomy and bilateral salpingo-oophorectomy. }\end{array}$} \\
\hline
\end{tabular}

Tab. 1. Clinicopathological features of ovarian serous cancer patients 


\begin{tabular}{|c|c|c|c|c|c|c|}
\hline \multirow[t]{2}{*}{$\begin{array}{l}\text { Univariate } \\
\text { analysis }\end{array}$} & \multicolumn{3}{|c|}{ OS } & \multicolumn{3}{|c|}{ DFS } \\
\hline & $p$ value & $\mathbf{O R}$ & $95 \% \mathrm{Cl}$ & $p$ value & $\mathbf{O R}$ & $95 \% \mathrm{Cl}$ \\
\hline Age & 0.70 & 1.00 & $0.98-7.01$ & 0.45 & 1.00 & 0.99-1.01 \\
\hline Stage & 0.00 & 1.85 & $1.52-2.26$ & 0.00 & 1.89 & $1.55-2.31$ \\
\hline Grade & 0.00 & 1.50 & 1.23-1.84 & 0.00 & 1.44 & 1.18-1.77 \\
\hline CA-125 & 0.00 & 1.82 & $1.33-2.49$ & 0.00 & 1.96 & $1.43-2.68$ \\
\hline Tumor size & 0.02 & 0.99 & $\begin{array}{c}0.990- \\
0.995 \\
\end{array}$ & 0.17 & 0.99 & $0.99-1.00$ \\
\hline Ascites & 0.00 & 1.85 & $1.34-2.54$ & 0.00 & 1.81 & 1.32-2.48 \\
\hline Optimality & 0.01 & 1.48 & $1.09-2.00$ & 0.00 & 1.67 & 1.23-2.27 \\
\hline NACT & 0.004 & 0.62 & $0.45-0.86$ & 0.005 & 0.63 & $0.46-0.87$ \\
\hline $\begin{array}{l}\text { Adjuvant } \\
\text { treatment }\end{array}$ & 0.003 & 0.11 & $1.03-1.20$ & 0.02 & 1.08 & $1.01-1.16$ \\
\hline Recurrence & 00 & 0.53 & 0.39 & . & 0.46 & $0.34-0.62$ \\
\hline \multirow[t]{2}{*}{$\begin{array}{l}\text { Multivariate } \\
\text { analysis }\end{array}$} & \multicolumn{3}{|c|}{ OS } & \multicolumn{3}{|c|}{ DFS } \\
\hline & $p$ value & $\mathbf{O R}$ & $95 \% \mathrm{Cl}$ & $p$ value & $\mathbf{O R}$ & $95 \% \mathrm{Cl}$ \\
\hline Age & 0.18 & 0.99 & $0.97-1.00$ & 0.25 & 0.99 & 0.97-1.00 \\
\hline Stage & 0.00 & 0.38 & $0.23-0.64$ & 0.00 & 0.39 & $0.23-0.65$ \\
\hline Grade & 0.01 & 1.33 & $1.07-1.65$ & 0.04 & 1.24 & $1.00-1.54$ \\
\hline CA-125 & 0.27 & 0.82 & $0.58-1.16$ & 0.14 & 0.76 & 0.54-1.09 \\
\hline Tumor size & 0.01 & 0.99 & $0.98-0.99$ & 0.36 & 0.99 & $0.99-1.00$ \\
\hline Ascites & 0.83 & 0.96 & $0.68-1.36$ & 0.94 & 0.98 & 0.69-1.40 \\
\hline Optimality & 0.003 & 0.57 & $0.40-0.82$ & 0.23 & 0.95 & 0.89-1.02 \\
\hline NACT & 0.02 & 0.66 & $0.47-0.94$ & 0.03 & 0.68 & $0.48-0.96$ \\
\hline $\begin{array}{l}\text { CT cycle } \\
\text { number }\end{array}$ & 0.001 & 1.11 & $1.04-1.19$ & 0.01 & 1.08 & $1.01-1.16$ \\
\hline Recurrence & 0.018 & 1.49 & $1.07-2.08$ & 0.002 & 1.66 & $1.20-2.29$ \\
\hline \multicolumn{7}{|c|}{$\begin{array}{l}\text { Cox regression analysis. } \\
\mathbf{9 5 \%} \text { Cl }-95 \% \text { confidence interval; CA-125 - cancer antigen 125; } \\
\text { CT - chemotherapy; DFS - disease-free survival; } \\
\text { NACT - neoadjuvant chemotherapy; 0S - overall survival. } \\
\text { Bold } p \text { values are }<0.05 \text { and statistically significant. } \\
\end{array}$} \\
\hline
\end{tabular}

Tab. 2. Factors affecting the OS and DFS in ovarian serous cancer patients stage 4 disease. Stage 4 patients received 3 cycles of NACT with platinum and taxane combinations before the operation. The mean CA-125 was $52.0 \pm 77.9$. A total of 34 patients (69.4\%) had LVSI. In 35 patients (71.4\%), the tumor size was $\geq 2 \mathrm{~cm}$. Nineteen patients $(38.8 \%)$ had cervical invasion. Overall, 33 patients (67.3\%) underwent optimal surgery. The mean pelvic lymph node number was $16.3 \pm 13.4$, and 8 patients $(16.3 \%)$ had pelvic lymph node metastases. The mean paraaortic lymph node number was $8.4 \pm 9.7$, and 6 patients (12.2\%) had paraaortic lymph node metastases. A total of 34 patients $(69.4 \%)$ received CT, while 38 patients $(77.6 \%)$ were treated by RT. In 11 patients $(22.4 \%)$, recurrence was detected (Tab. 4). In univariate analysis, disease stage, optimality, RT and recurrence had impact on the OS and DFS. In multivariate analysis, only optimality had an effect on the DFS ( $p=0.04)$ (Tab. 5). In binary logistic regression analysis, optimality was the only significant prognostic factor for recurrence ( $p=0.01$, odds ratio: 0.06 ) (Tab. 6).

\section{DISCUSSION}

In this study, we categorized cases with the serous type of ovarian cancers and uterine cancers according to their descriptives features and prognostic factors that affected patient survival and recurrence. We demonstrated several statistically significant independent factors determining survival and recurrence in ovarian serous cancer patients, and only one statistically significant independent factor affecting survival and recurrence in uterine serous cancer patients. To our knowledge, this study is the only one in the literature that identified prognostic factors for survival and recurrence both in patients diagnosed with ovarian and uterine serous carcinoma.

Ovarian cancers are a heterogenous group of malignancies differentiated by the origin of cells, pathological grade, risk factors, prognosis, and treatment ${ }^{(6)}$. Epithelial cancers are classified by tumor cell histology as serous (52\%), endometrioid $(10 \%)$, mucinous $(6 \%)$, or clear cell $(6 \%)$,

\begin{tabular}{|l|c|c|c|c|c|c|c|}
\hline & B & SE & Wald & df & Exp(B) & 95\% CI & $\boldsymbol{p}$ \\
\hline Age & 0.012 & 0.011 & 1.15 & 1 & 1.01 & $0.99-1.03$ & 0.282 \\
\hline Stage & 1.07 & 0.35 & 9.09 & 1 & 2.92 & $1.45-5.86$ & $\mathbf{0 . 0 0 3}$ \\
\hline Grade & -0.11 & 0.16 & 0.51 & 1 & 0.88 & $0.64-1.22$ & 0.472 \\
\hline CA-125 & 0.17 & 0.26 & 0.43 & 1 & 1.18 & $0.71-1.97$ & 0.512 \\
\hline Tumor size & -0.002 & 0.00 & 0.32 & 1 & 0.99 & $0.99-1.00$ & 0.572 \\
\hline Ascites & -0.058 & 0.26 & 0.04 & 1 & 0.94 & $0.56-1.58$ & 0.827 \\
\hline Suboptimality & 0.91 & 0.27 & 11.1 & 1 & 2.49 & $1.45-4.26$ & $\mathbf{0 . 0 0 1}$ \\
\hline NACT & 0.18 & 0.26 & 0.46 & 1 & 1.20 & $0.70-2.03$ & 0.494 \\
\hline CT cycle number & -0.029 & 0.05 & 0.27 & 1 & 0.97 & $0.86-1.08$ & 0.603 \\
\hline Constant & -1.23 & 0.77 & 2.57 & 1 & 0.29 & \\
\hline CA-125 - cancer antigen 125; CT - chemotherapy; NACT - neodjuvant chemotherapy. & & & \\
\hline
\end{tabular}

Tab. 3. Binary logistic regression analyses of ovarian serous cancer patients (Dependent Factor: Recurrence, Model = Enter; Hosmer and Lemeshow Test: 0.63) 


\begin{tabular}{|c|c|c|}
\hline Stage & $n(\%)$ & $95 \% \mathrm{Cl}$ \\
\hline 1 & $26(53.1 \%)$ & $38.5-67.4$ \\
\hline 2 & $6(12.2 \%)$ & $4.0-22.0$ \\
\hline 3 & $10(20.4 \%)$ & $9.8-31.7$ \\
\hline 4 & $7(14.3 \%)$ & $5.6-25.0$ \\
\hline \multirow[t]{2}{*}{ Age } & Mean & $\pm S D$ \\
\hline & 67.2 & 10.6 \\
\hline \multirow[t]{2}{*}{ CA-125 } & Mean & $\pm S D$ \\
\hline & 52.0 & 77.9 \\
\hline \multirow[t]{2}{*}{ NACT } & $n(\%)$ & $95 \% \mathrm{Cl}$ \\
\hline & $7(14.3 \%)$ & $5.6-25.0$ \\
\hline Tumor size & $n(\%)$ & $95 \% \mathrm{Cl}$ \\
\hline$<2 \mathrm{~cm}$ & $14(28.6 \%)$ & $15.7-41.5$ \\
\hline$\geq 2 \mathrm{~cm}$ & $35(71.4 \%)$ & $58.5-84.3$ \\
\hline LVSI & $n(\%)$ & $95 \% \mathrm{Cl}$ \\
\hline No & $15(30.6 \%)$ & $17.8-45.1$ \\
\hline Yes & $34(69.4 \%)$ & $54.9-82.2$ \\
\hline OP & $n(\%)$ & $95 \% \mathrm{Cl}$ \\
\hline TAH BSO & $3(6.1 \%)$ & $0.0-12.2$ \\
\hline TAH BSO PLND & $7(14.3 \%)$ & $6.1-24.5$ \\
\hline TAH BSO PPLND & $39(79.6 \%)$ & $69.4-89.8$ \\
\hline Optimality & $n(\%)$ & $95 \% \mathrm{Cl}$ \\
\hline Optimal & $16(32.7 \%)$ & $20.0-46.6$ \\
\hline Suboptimal & $33(67.3 \%)$ & $53.4-80.0$ \\
\hline \multicolumn{3}{|l|}{ MI } \\
\hline No & $17(34.7 \%)$ & $21.7-48.9$ \\
\hline Yes & $32(65.3 \%)$ & $51.1-78.3$ \\
\hline CXI & $n(\%)$ & $95 \% \mathrm{Cl}$ \\
\hline No & $30(61.2 \%)$ & $48.0-74.5$ \\
\hline Yes & $19(38.8 \%)$ & $25.5-52.0$ \\
\hline Recurrence & $n(\%)$ & $95 \% \mathrm{Cl}$ \\
\hline No & $11(22.4 \%)$ & $10.9-34.6$ \\
\hline Yes & $38(77.6 \%)$ & $65.4-89.1$ \\
\hline CT & $n(\%)$ & $95 \% \mathrm{Cl}$ \\
\hline No & $15(30.6 \%)$ & $18.2-44.0$ \\
\hline Yes & $34(69.4 \%)$ & $56.0-81.8$ \\
\hline RT & $n(\%)$ & $95 \% \mathrm{Cl}$ \\
\hline No & $11(22.4 \%)$ & $10.9-34.6$ \\
\hline Yes & $38(77.6 \%)$ & $65.4-89.1$ \\
\hline \multirow[t]{2}{*}{ Pelvic lymph node number } & Mean & $\pm S D$ \\
\hline & 16.33 & 13.4 \\
\hline Pelvic lymph node metastasis & $n(\%)$ & $95 \% \mathrm{Cl}$ \\
\hline No & $41(83.7 \%)$ & $72.5-93.6$ \\
\hline Yes & 8 & $6.4-27.5$ \\
\hline \multirow[t]{2}{*}{ Paraaortic lymph node number } & Mean & $\pm S D$ \\
\hline & 8.4 & 9.7 \\
\hline
\end{tabular}

Tab. 4. Clinicopathological features of uterine serous cancer patients

\begin{tabular}{|l|c|c|}
\hline $\begin{array}{l}\text { Paraaortic lymph node } \\
\text { metastasis }\end{array}$ & $\boldsymbol{n}(\%)$ & $\mathbf{9 5 \%} \mathrm{Cl}$ \\
\hline No & $43(87.8 \%)$ & $78.3-95.8$ \\
\hline Yes & $6(12.2 \%)$ & $0.0-37.4$ \\
\hline
\end{tabular}

95\% Cl - 95\% confidence interval; CA-125 - cancer antigen 125;

CT - chemotherapy; CXI - cervix invasion; LVSI - lymphovascular space invasion; $\mathbf{M I}$ - myometrial invasion; NACT - neoadjuvant chemotherapy; OP - operation; PLND - pelvic lymph node dissection; PPLND - pelvic paraaortic lymph npde dissection; RT - radiotherapy; TAH BSO - total abdominal hysterectomy and bilateral salpingo-oophorectomy.

Tab. 4. Clinicopathological features of uterine serous cancer patients (cont.)

with one-quarter being more rare subtypes or unspecified ${ }^{(7)}$. In this study, serous cancer type was seen in $46.3 \%$ of all epithelial ovarian cancer patients. Type II epithelial cancers are high grade and characterized by the involvement of both ovaries, aggressive behavior, late stage at diagnosis, and low survival rates. They are thought to originate as fallopian tube fimbriae carcinomas that spread to the ovaries and/or the peritoneum ${ }^{(8)}$. Type II cancers are primarily high-grade serous carcinomas, the most common epithelial subtype, but they also include carcinosarcomas and undifferentiated carcinomas $^{(8)}$. Similarly in this study, $79.5 \%$ of serous ovarian carcinomas were grade 2 and 3. Patient age is widely accepted as a prognostic factor for ovarian cancer survival ${ }^{(9,10)}$. Older women have an increased risk of treatment failure and hence higher rates of recurrences and lower survival rates ${ }^{(11)}$. In contrast, in the present study patient age had no significant effect on survival and recurrence in Cox regression and logistic regression analysis.

Serum CA-125 levels, also accepted as a predictive and prognostic factor for ovarian cancers, are used for monitoring response after treatment and patient survival. Serum levels of CA-125 measured before any treatment have been evaluated in epithelial ovarian cancer as a predictor of patient survival; however, the results in survival index are controversial, as CA-125 levels are influenced by several variables. Morales-Vásquez et al. reported an enhanced survival in patients with high-grade serous carcinoma, FIGO stage III, and pretreatment serum CA-125 levels higher than $500 \mathrm{U} / \mathrm{mL}^{(12)}$. In the present study, CA-125 values were significant for the OS and DFS in univariate Cox regression analysis but not in multivariate analysis.

Dao et al. reported that long-term survivors of high-grade serous carcinoma patients have high surgical resection to no visible disease ${ }^{(13)}$. In the present study, optimal surgery was significant for the OS and DFS in both univariate and multivariate analyses. The National Comprehensive Cancer Network guidelines recommend at least a total of $6 \mathrm{cy}$ cles of CT in suspected cases of unresectable advanced-stage ovarian cancer, as well as interval debulking surgery (IDS) prior to the fourth cycle of $\mathrm{NACT}^{(14)}$. In one study, it was indicated that completing at least 6 cycles of total CT was an independent prognostic factor for survival in patients undergoing NACT, IDS and postoperative adjuvant CT. 


\begin{tabular}{|c|c|c|c|c|c|c|}
\hline \multirow{2}{*}{ Univariate analysis } & \multicolumn{3}{|c|}{ OS } & \multicolumn{3}{|c|}{ DFS } \\
\hline & $p$ value & OR & $95 \% \mathrm{Cl}$ & $p$ value & OR & $95 \% \mathrm{Cl}$ \\
\hline Stage & 0.01 & 1.69 & $1.09-2.61$ & 0.04 & 1.57 & $1.00-2.46$ \\
\hline Optimality & 0.01 & 0.35 & $0.15-0.85$ & 0.00 & 0.29 & $0.12-0.69$ \\
\hline CA-125 & 0.12 & 1.00 & $0.99-1.00$ & 0.17 & 1.00 & $0.99-1.00$ \\
\hline LVSI & 0.39 & 1.48 & $0.59-3.66$ & 0.43 & 1.44 & $0.57-3.62$ \\
\hline Tumor size & 0.94 & 1.03 & $0.41-2.58$ & 0.94 & 0.96 & $0.38-2.42$ \\
\hline $\mathrm{Ml}$ & 0.68 & 0.83 & $0.34-2.00$ & 0.92 & 0.96 & $0.40-2.30$ \\
\hline CXI & 0.54 & 0.73 & $0.27-1.98$ & 0.61 & 0.76 & $0.26-2.18$ \\
\hline Pelvic metastasis & 0.46 & 1.09 & $0.85-1.40$ & 0.88 & 0.98 & $0.77-1.24$ \\
\hline Paraaortic metastasis & 0.90 & 0.98 & $0.72-1.33$ & 0.58 & 0.90 & $0.62-1.30$ \\
\hline $\mathrm{RT}$ & 0.006 & 0.26 & $0.09-0.68$ & 0.014 & 0.29 & $0.11-0.77$ \\
\hline $\mathrm{CT}$ & 0.97 & 0.98 & $0.41-2.34$ & 0.84 & 0.91 & $0.37-2.21$ \\
\hline Recurrence & 0.12 & 2.08 & $0.82-5.27$ & 0.004 & 4.36 & $1.61-11.7$ \\
\hline \multirow{2}{*}{ Multivariate analysis } & \multicolumn{3}{|c|}{ OS } & \multicolumn{3}{|c|}{ DFS } \\
\hline & $p$ value & OR & $95 \% \mathrm{Cl}$ & $p$ value & OR & $95 \% \mathrm{Cl}$ \\
\hline Stage & 0.98 & 1.01 & $0.34-2.94$ & 0.96 & 1.02 & $0.35-2.95$ \\
\hline Optimality & 0.061 & 0.34 & $0.11-1.04$ & 0.04 & 3.26 & $1.05-10.0$ \\
\hline CA-125 & 0.45 & 1.00 & $0.99-1.01$ & 0.72 & 1.00 & $0.99-1.01$ \\
\hline LVSI & 0.32 & 0.52 & $0.15-1.86$ & 0.43 & 0.61 & $0.17-2.10$ \\
\hline Tumor size & 0.95 & 0.96 & $0.28-3.25$ & 0.61 & 0.73 & $0.21-2.48$ \\
\hline $\mathrm{Ml}$ & 0.65 & 0.74 & $0.20-2.68$ & 0.61 & 0.71 & $0.18-2.71$ \\
\hline CXI & 0.93 & 0.92 & $0.11-7.57$ & 0.75 & 0.72 & $0.09-5.50$ \\
\hline Pelvic metastasis & 0.35 & 1.46 & $0.65-3.30$ & 0.43 & 1.43 & $0.58-3.47$ \\
\hline Paraaortic metastasis & 0.56 & 0.60 & $0.11-3.35$ & 0.46 & 0.50 & $0.08-3.18$ \\
\hline RT & 0.29 & 2.63 & $0.43-15.7$ & 0.19 & 3.26 & $0.55-19.1$ \\
\hline $\mathrm{CT}$ & 0.18 & 2.59 & $0.64-10.4$ & 0.14 & 2.64 & $0.70-9.87$ \\
\hline Recurrence & 0.72 & 1.28 & $0.31-5.33$ & 0.10 & 2.81 & $0.80-9.90$ \\
\hline \multicolumn{7}{|c|}{$\begin{array}{l}\text { Cox regression analysis. } \\
\mathbf{9 5 \%} \text { CI - 95\% confidence interval; CA-125 - cancer antigen 125; CT - chemotherapy; CXI - cervix invasion; DFS - disease-free survival; LVSI - lymphovascular space } \\
\text { invasion; } \mathbf{M I} \text { - myometrial invasion; NACT - neoadjuvant chemotherapy; OS - overall survival; RT - radiotherapy. } \\
\text { Bold } p \text { values are < }<0.05 \text { and statistically significant. }\end{array}$} \\
\hline
\end{tabular}

Tab. 5. Factors affecting the overall and disease-free survival in uterine serous cancer patients

In our study, $28.3 \%(n=109)$ patients received NACT, and NACT had a statistically significant effect both on the OS and DFS, but no impact on recurrence ${ }^{(15)}$. Despite a combination of cytoreductive surgery and CT treatment, the majority of patients develop recurrent disease leading to 5-year survival rates of around $30 \%{ }^{(16)}$. The risk factors for recurrence

\begin{tabular}{|l|c|c|c|c|c|c|c|}
\hline & $\mathbf{B}$ & SE & Wald & df & Exp(B) & $\mathbf{9 5} \% \mathbf{C I}$ & $\boldsymbol{p}$ \\
\hline Age & 0.05 & 0.047 & 1.25 & 1 & 0.26 & $0.96-1.15$ & 1.05 \\
\hline Optimality & -2.81 & 1.17 & 5.77 & 1 & $\mathbf{0 . 0 1}$ & $0.00-0.59$ & 0.06 \\
\hline Stage & -2.35 & 1.27 & 3.40 & 1 & 0.06 & $0.00-1.15$ & 0.09 \\
\hline CA-125 & 0.017 & 0.009 & 4.05 & 1 & 0.04 & $1.00-1.03$ & 1.01 \\
\hline LVSI & 2.31 & 1.78 & 1.68 & 1 & 1.19 & $0.30-335.7$ & 10.1 \\
\hline Tumor size & -0.55 & 1.11 & 0.25 & 1 & 0.61 & $0.06-5.09$ & 0.57 \\
\hline MI & -1.73 & 1.38 & 1.57 & 1 & 0.20 & $0.01-2.64$ & 0.17 \\
\hline CXI & 2.8 & 1.81 & 2.43 & 1 & 0.11 & $0.48-594.7$ & 16.9 \\
\hline CT & 2.69 & 1.82 & 2.17 & 1 & 0.14 & $0.41-532.9$ & 14.8 \\
\hline RT & -3.01 & 1.96 & 2.36 & 1 & 0.12 & $0.00-2.28$ & $\mathbf{0 . 0 4}$ \\
\hline Constant & -0.88 & 3.45 & 0.066 & 1 & 0.79 & & 0.41 \\
\hline CA-125 - cancer antigen 125; CT - chemotherapy; CXI - cervix invasion; LVI - lymphovascular space invasion; MI - myometrial invasion; RT - radiotherapy.
\end{tabular}

Tab. 6. Binary logistic regression analyses of uterine serous cancer patients (Dependent Factor: Recurrence, Model = Enter; Hosmer and Lemeshow Test: 0.72) 
include advanced disease, suboptimal surgery, response to CT, tumor grade, and histological tumor type $\mathrm{e}^{(17)}$. In this study, for serous ovarian cancer patients, recurrence had an effect on survival in univariate and multivariate Cox regression analyses, whereas for recurrence development, suboptimality and advance stages represented statistically significant prognostic factors in logistic regression analysis. In a study of low-grade serous ovarian carcinomas, it was found that advanced stages (stage 3,4), LVSI, and suboptimal surgery were independent factors for decreased DFS, while suboptimal surgery was independently associated with decreased OS. In this study, LVSI was also associated with lymphatic recurrences ${ }^{(18)}$.

Uterine serous carcinoma (USC) is the most common nonendometrioid subtype of endometrial cancer, accounting for approximately $10 \%$ of all endometrial cancer cases. Typically diagnosed at an advanced stage, USC is an aggressive histologic subtype associated with a disproportionate number (39\%) of endometrial cancer deaths ${ }^{(19)}$. In this study, USC accounted $2.4 \%$ of all uterine carcinomas. The median age group of patients with endometrial carcinoma is 61 years, with $75-80 \%$ of affected women being postmenopausal. In this study, the mean age of serous uterine carcinoma patients was $67.2 \pm 10.6$ years. Endometrial cancer is often detected at an early stage because it frequently produces abnormal vaginal bleeding. In this study, $53.1 \%$ of the patients were at stage 1. Tejerizo-García et al. reported that FIGO stage and tumor grade were independent prognostic factors of diseasefree survival and overall survival in endometrial cancer patients ${ }^{(20)}$. Similarly, for USC patients we identified stage as a significant independent factor for the DFS and OS in univariate Cox regression analysis. However, it was not a prognostic factor for the OS and DFS in multivariate Cox regression analysis. Optimality was demonstrated as the main independent prognostic factor for the DFS and OS in Cox regression analysis in USC patients. The recurrence rate of USC is high, estimated to be $31-80 \%$ even in its early stages ( 1 and 2$)^{(21)}$. In this study, $22.4 \%$ of USC patients had recurrence, and recurrence had an effect on survival in univariate Cox regression analysis. In logistic regression analysis, only optimality was an independent significant factor for recurrence. In a multicenter uterine serous cancer study reported by Zhong et al., advanced stage, myometrial invasion, adnexal involvement, lymph node metastasis, and positive peritoneal cytology were stated as independently associated prognostic factors for the DFS, and these listed factors were also associated with disease recurrence ${ }^{(22)}$. However, in a single institution study of 62 uterine serous carcinoma cases it was found that positive peritoneal cytology, positive LVSI, positive lymph nodes, and adjuvant therapies were not statistically significant in survival analyses ${ }^{(23)}$.

In conclusion, disease stage, grade, optimality, NACT, and adjuvant CT cycle number had impact on overall and disease-free survival in ovarian serous cancer patients in both univariate and multivariate Cox regression analyses, where- prognostic factors for recurrence in ovarian serous cancer patients. However, in patients with uterine serous carcinomas, optimal surgery was the only independent prognostic factor both for survival and recurrence.

\section{Conflict of interest}

We have no conflict of interest. I have no financial relationship with the organization that sponsored the research, and I had full control of all primary data. I agree to allow the Journal to review their data, if requested.

\section{References}

1. Ferlay J, Soerjomataram I, Dikshit R et al.: Cancer incidence and mortality worldwide: sources, methods and major patterns in GLOBOCAN 2012. Int J Cancer 2015; 136: E359-E386.

2. Bowtell DD: The genesis and evolution of high-grade serous ovarian cancer. Nat Rev Cancer 2010; 10: 803-808.

3. Hennessy BT, Coleman RL, Markman M: Ovarian cancer. Lancet 2009; 374: 1371-1382.

4. Ozols RF, Bundy BN, Greer BE et al.; Gynecologic Oncology Group: Phase III trial of carboplatin and paclitaxel compared with cisplatin and paclitaxel in patients with optimally resected stage III ovarian cancer: a Gynecologic Oncology Group study. J Clin Oncol 2003; 21: 3194-3200.

5. Armstrong DK, Bundy B, Wenzel L et al.; Gynecologic Oncology Group: Intraperitoneal cisplatin and paclitaxel in ovarian cancer. N Engl J Med 2006; 354: 34-43.

6. Wentzensen N, Poole EM, Trabert B et al.: Ovarian cancer risk factors by histologic subtype: An analysis from the Ovarian Cancer Cohort Consortium. J Clin Oncol 2016; 34: 2888-2898.

7. SEER ${ }^{\star}$ Stat Database: NAACCR Incidence Data - CiNA Analytic File, 1995-2014, for Expanded Races, Custom File With County, ACS Facts and Figures projection Project [which includes data from CDC's National Program of Cancer Registries (NPCR), CCCR's Provincial and Territorial Registries, and the NCI's Surveillance, Epidemiology and End Results (SEER) Registries], certified by the North American Association of Central Cancer Registries (NAACCR) as meeting high-quality incidence data standards for the specified time periods, submitted December 2016.

8. Kurman RJ, Shih IeM: The dualistic model of ovarian carcinogenesis: revisited, revised, and expanded. Am J Pathol 2016; 186 : 733-747.

9. Hainsworth JD, Grosh WW, Burnett LS et al.: Advanced ovarian cancer: long-term results of treatment with intensive cisplatinbased chemotherapy of brief duration. Ann Intern Med 1988; 108: 165-170.

10. Neijt JP, ten Bokkel Huinink WW, van der Burg ME et al.: Longterm survival in ovarian cancer. Mature data from The Netherlands Joint Study Group for Ovarian Cancer. Eur J Cancer 1991; 27: 1367-1372.

11. Winter WE $3^{\text {rd }}$, Maxwell GL, Tian C et al.; Gynecologic Oncology Group Study: Prognostic factors for stage III epithelial ovarian cancer: a Gynecologic Oncology Group Study. J Clin Oncol 2007; 25: 3621-3627.

12. Morales-Vásquez F, Pedernera E, Reynaga-Obregón J et al.: High levels of pretreatment CA125 are associated to improved survival in high grade serous ovarian carcinoma. J Ovarian Res 2016; 9: 41.

13. Dao F, Schlappe BA, Tseng J et al.: Characteristics of 10-year survivors of high-grade serous ovarian carcinoma. Gynecol Oncol 2016; 141: 260-263.

14. National Comprehensive Cancer Network: NCCN Clinical Practice Guidelines in Oncology (NCCN Guidelines ${ }^{\varpi}$ ): Ovarian cancer including fallopian tube cancer and primary peritoneal cancer. Washington 2017. 
15. Chung YS, Kim YJ, Lee I et al.: Impact of neoadjuvant chemotherapy and postoperative adjuvant chemotherapy cycles on survival of patients with advanced-stage ovarian cancer. PLoS One 2017; 12: e0183754.

16. Bristow RE, Tomacruz RS, Armstrong DK et al.: Survival effect of maximal cytoreductive surgery for advanced ovarian carcinoma during the platinum era: a meta-analysis. J Clin Oncol 2002; 20: 1248-1259.

17. Vergote I, Tropé CG, Amant $\mathrm{F}$ et al.: Neoadjuvant chemotherapy or primary surgery in stage IIIC or IV ovarian cancer. N Engl J Med 2010; 363: 943-953.

18. Matsuo K, Wong KK, Fotopoulou C et al.: Impact of lympho-vascular space invasion on tumor characteristics and survival outcome of women with low-grade serous ovarian carcinoma. J Surg Oncol 2018; 117: 236-244.
19. Hamilton CA, Cheung MK, Osann K et al.: Uterine papillary serous and clear cell carcinomas predict for poorer survival compared to grade 3 endometrioid corpus cancers. Br J Cancer 2006; 94: 642-646.

20. Tejerizo-García A, Jiménez-López JS, Muñoz-González JL et al.: Overall survival and disease-free survival in endometrial cancer: prognostic factors in 276 patients. Onco Targets Ther 2013; 9: 1305-1313.

21. del Carmen MG, Birrer M, Schorge JO: Uterine papillary serous cancer: a review of the literature. Gynecol Oncol 2012; 127: 651-661.

22. Zhong $\mathrm{X}$, Wang J, Kaku $\mathrm{T}$ et al.: Prognostic factors of uterine serous carcinoma - a multicenter study. Int J Gynecol Cancer 2018; 28: 1138-1144.

23. Black $\mathrm{C}$, Feng $\mathrm{A}$, Bittinger $\mathrm{S}$ et al.: Uterine papillary serous carcinoma: a single-institution review of 62 cases. Int J Gynecol Cancer 2016; 26: 133-140. 